\title{
BCL-2 Inhibition in the Treatment of Multiple Myeloma
}

\author{
Gabor Mikala* \\ Department of Haematology and Stem Cell Transplantation South-Pest Central Hospital, National Institute for Haematoloy and Infectious Diseases, Hungary
}

Received: May 11, 2018; Published: May 18, 2018

*Corresponding author: Gabor Mikala, Department of Haematology and Stem Cell Transplantation South-Pest Central Hospital, National Institute for Haematology and Infectious Diseases, Budapest, Hungary, H-1097

\section{Introduction}

Multiple myeloma is the second most common haematological malignancy - though multiple effective therapeutic options exist for disease control - cure of myeloma still remains the exception. Our present therapeutic approach is founded on apoptosis induction in tumor cells by various different stimuli, most commonly by DNA damage, oxidative or endoplasmic reticular (proteomic) stress. Effective treatment of relapsed and/or refractory disease rarely provides long term disease control mainly due to development of resistance to pharmacological induction of apoptosis pathways.

As members of the B-cell lymphoma 2 (BCL-2) family of mitochondrial proteins are crucial regulators of common apoptotic pathways, inhibition of anti-apoptotic members of this protein family has gained acceptance in the clinical practice [1]. This is especially true in the treatment of chronic lymphocytic leukemia (CLL), hallmarked by the approval of the BCL-2 inhibitor venetoclax as a particularly effective salvage therapy for this disease [2]. Proteins of the BCL-2 family are commonly categorized as proor anti-apoptotic members, the latter being BCL-2, BCL-XL, MCL1 and BCL-W [3]. The cell death mediator proteins BAK and BAX facilitate apoptosis by induction of mitochondrial depolarization, cytochrome c leakage and finally caspase-dependent cell death. The crucial function of anti-apoptotic BCL-2 family members is the inhibition of BAK/BAX activity through direct protein-protein interactions.

While CLL cells may effectively evade apoptosis by pronounced reliance on BCL-2 activity, in other malignancies distinct BCL-2 family members may have a greater influence. As prior preclinical studies indicated that in plasma cells as well as in multiple myeloma MCL-1 may be the major anti-apoptotic BCL-2 analogue [4], clinical testing of the BCL-2 specific venetoclax (ABT-199) in this disease started with a moderate level of enthusiasm. In the Phase I clinical study M13-367, venetoclax monotherapy was given to relapsed/refractory multiple myeloma patients with measurable disease (with a median of 5 prior lines of therapy). $1200 \mathrm{mg}$ of venetoclax was determined as a tolerated, safe (and effective) dose $[5,6]$. During the trial, the preclinical hypothesis [7] was confirmed that myeloma patients with translocation $\mathrm{t}(11 ; 14)(\mathrm{IgH} /$ CyclinD1) responded more favorably to this therapy.

Therefore, this specific cohort was expanded to include 30 patients, a feasible approach as approximately $20 \%$ of all myeloma patients harbor this translocation. With respect to the entire study population (66 patients), the objective response rate was $21 \%$, however, nearly all of the responding patients came from the $\mathrm{t}(11 ; 14)$ subgroup. In this subgroup, the response rate amounted to $40 \%$ that included $14 \%$ complete remissions. Looking in the reverse, only 2 of those patients without translocation $t(11 ; 14)$ in the entire trial population of 66 individuals had any meaningful response to venetoclax. This observation coincides with the preclinical experimental finding that in case of translocation $t(11 ; 14)$ in multiple myeloma, the ratio of BCL-2 to MCL-1 expression is shifted towards higher BCL-2 levels $[7,8]$.

It was also observed that venetoclax-sensitive cells exhibited not only higher BCL-2 expression but venetoclax-resistant cells have higher BCL-XL and MCL-1 expression. If you consider venetoclax a targeted therapy for multiple myeloma with translocation $\mathrm{t}(11 ; 14)$ and make a naive comparison with other available therapeutic options for a heavily pretreated relapsed-refractory patient population, judicial use of venetoclax may emerge as a particularly effective salvage option for these patients. $40 \%$ overall response rate (ORR) for venetoclax monotherapy compares favorably to $31 \%$ ORR for pomalidomide/dexamethason (from trial MM-003) [9], 29\% ORR for daratumumab (from trial SIRIUS) [10] and $24 \%$ ORR for carfilzomib (from trial PX-171-003) [11]. The particular effectiveness of venetoclax in this genetic subpopulation is underlined by the $27 \%>$ VGPR (very good partial remission) rate, quite unprecedented in this severely pretreated population, and importantly independent of refractory status to prior therapies. Since this high efficacy of venetoclax is specifically limited to patients with translocation $t(11 ; 14)$, and even here the response 
is not uniform, BLC-2 protein profiling may be the best option for selecting the right patients for this targeted therapy.

In selected myeloma patients with translocation $\mathrm{t}(11 ; 14)$ and high BCL-2: BCL-XL ratio the overall response rate may amount to $88 \%$ with CR rate of $44 \%$, that is really exceptional in multiple myeloma therapy. As an added bonus, duration of response in this subgroup of high BCL-2: BCL-XL ratio had a median of 12 months. Validity of these early trial observations was further supported by individual case reports of myeloma and plasma cell leukemia patients in dire clinical situation responding exceptionally well to venetoclax administration $[12,13]$. Combination of BCL-2 inhibition with other therapeutic modalities may be an option to widen the range of applicability of this approach. Preliminary experimental results indicated that the proteasome inhibitor bortezomib, a highly effective myeloma drug may exert some of its activity by stabilizing the MCL-1 neutralizing protein NOXA [14].

As MCL-1 expression may be a potential resistance mechanism to venetoclax activity, bortezomib may prove to be a good combination partner to venetoclax. It was also shown that dexamethason sensitizes myeloma cell lines and primary myeloma cells to venetoclax activity, therefore clinical study M12-901 was initiated to test this triple combination (bortezomib-venetoclaxdexamethason) in relapsed/refractory myeloma patients [15]. Here, the objective response level reached $67 \%$ and was not restricted at all to $\mathrm{t}(11 ; 14)$ patients, proving the validity of the approach. Moreover, for those patients not refractory to proteasome inhibitors and immunomodulatory agents, the response rate for the triple combination reached an impressive 92\%. Overall response rates were similar across different cytogenetic profiles; most notably it was $47 \%$ in those patients [15] who had deletion $17 p$, a particularly high-risk subgroup with severely compromised apoptosis process. The importance of high BCL-2 expression predicting better and more prolonged clinical response was also underlined in this study. Higher baseline BCL-2 levels were detected in patients that achieved a PR or better versus those who did not. Importantly, higher BCL-2 levels were seen in the patient subgroup that had only 1-3 prior lines of therapy compared to those with more than 4 lines of therapy. Median time to progression (11.6 months vs 5.7 months) was longer in patients with high versus low BCL-2 expression. These very promising early phase data prompted the initiation of the M14-031 (Bellini) clinical trial that aims for registration of the bortezomib-venetoclax-dexamethason triple combination as salvage therapy for relapsed/refractory multiple myeloma with 1-3 prior lines of therapy.

Based on these impressive early clinical data, it is conceivable that BCL-2 inhibition by venetoclax has just provided us the entry point of individualized targeted therapy for a certain important subset of multiple myeloma patients. As newer and newer apoptosis inducers, including MCL-1 inhibitors are entering the clinic, hopefully many of our patients may soon enjoy the benefit of personalized myeloma treatment approaches.

\section{References}

1. Khan N, Kahl B (2018) Targeting BCL-2 in Hematologic Malignancies. Target Oncol.

2. Gentile M, Petrungaro A, Uccello G, Vigna E, Recchia AG, Caruso N, et al. (2017) Venetoclax for the treatment of chronic lymphocytic leukemia. Expert OpinInvestig Drugs 26(11): 1307-1316.

3. Kale J, Osterlund EJ, Andrews DW (2018) BCL-2 family proteins: changing partners in the dance towards death. Cell Death Differ 25(1): 65-80.

4. Gomez-Bougie P, Amiot M (2013) Apoptotic machinery diversity in multiple myeloma molecular subtypes. Front Immunol 4: 467.

5. Kumar S, Vij R, Kaufman JL, Mikhael J, Facon T, et al. (2016) Venetoclax Monotherapy for Relapsed/Refractory Multiple Myeloma: Safety and Efficacy Results from a Phase I Study. Blood 128: 488.

6. Kumar S, Kaufman JL, Gasparetto C, Mikhael J, Vij R, et al. (2017) Efficacy of venetoclax as targeted therapy for relapsed/refractory $\mathrm{t}(11 ; 14)$ multiple myeloma. Blood 130(22): 2401-2409.

7. Touzeau C, Dousset C, Le Gouill S, Sampath D, Leverson JD, et al. (2014) The Bcl-2 specific BH3 mimetic ABT-199: a promising targeted therapy for $\mathrm{t}(11 ; 14)$ multiple myeloma. Leukemia 28(1): 210-212.

8. Punnoose EA, Leverson JD, Peale F, Boghaert ER, Belmont LD, et al. (2016) Expression Profile of BCL-2, BCL-XL, and MCL-1 Predicts Pharmacological Response to the BCL-2 Selective Antagonist Venetoclax in Multiple Myeloma Models. Mol Cancer Ther 15(5): 1132-1144.

9. Miguel JS, Weisel K, Moreau P, Lacy M, Song K, et al. (2013) Pomalidomide plus low-dose dexamethasone versus high-dose dexamethasone alone for patients with relapsed and refractory multiple myeloma (MM-003): a randomised, open-label, phase 3 trial. Lancet Oncol 14(11): 1055-1066.

10. Lonial S, Weiss BM, Usmani SZ, Singhal S, Chari A, et al. (2016) Daratumumab monotherapy in patients with treatment-refractory multiple myeloma (SIRIUS): an open-label, randomised, phase 2 trial. Lancet 387(10027): 1551-1560.

11. Siegel DS, Martin T, Wang M, Vij R, Jakubowiak AJ, et al. (2012) A phase 2 study of single-agent carfilzomib (PX-171-003-A1) in patients with relapsed and refractory multiple myeloma. Blood 120(14): 2817-2825.

12. TouzeauC, Le Gouill S, Mahé B, Boudreault JS, Gastinne T, et al. (2016) Deep and sustained response after venetoclax therapy in a patient with very advanced refractory myeloma with translocation $t(11 ; 14)$. Haematologica 102(3): e112-e114.

13. Gonsalves WI, Buadi FK, Kumar SK (2018) Combination therapy incorporating $\mathrm{Bcl}-2$ inhibition with Venetoclax for the treatment of refractory primary plasma cell leukemia with $\mathrm{t}(11 ; 14)$. Eur J Haematol $100(2): 215-217$.

14. Matulis SM, Gupta VA, Nooka AK, Hollen HV, Kaufman JL, et al. (2016) Dexamethasone treatment promotes Bcl-2 dependence in multiple myeloma resulting in sensitivity to venetoclax. Leukemia 30(5): 10861093.

15. Moreau P, Chanan-Khan A, Roberts AW, Agarwal AB, Facon T, et al. (2017) Promising efficacy and acceptable safety of venetoclax plus bortezomib and dexamethasone in relapsed/refractory MM. Blood 130(22): 23922400 . 
(C) (i) This work is licensed under Creative

Submission Link: https://biomedres.us/submit-manuscript.php

\begin{tabular}{ll}
\hline & Assets of Publishing with us \\
BIOMEDICAL & Global archiving of articles \\
RESERCHES & - Immediate, unrestricted online access \\
& - Rigorous Peer Review Process \\
\hline issN:2574-1241 & - Authors Retain Copyrights \\
\hline
\end{tabular}

